\title{
A New Genus, Two New Species, and a New Generic Name of Phyllocop- tine Mites (Acari: Eriophyidae) Infesting Yang-na, Dipterocarpus alatus Roxb. ex G. Don (Dipterocarpaceae) in Thailand
}

\author{
Angsumarn CHANDRAPATYA ${ }^{1}$, Ploychompoo KONVIPASRUANG $^{2}$ and \\ Jim W. AMRINE, Jr. ${ }^{3}$ \\ ${ }^{I}$ Department of Entomology, Kasetsart University, Chatuchak, Bangkok 10900, Thailand \\ ${ }^{2}$ Plant Protection Research and Development Office, Department of Agriculture, Phaholyothin \\ Rd., Chatuchak, Bangkok 10900, Thailand \\ ${ }^{3}$ Division of Plant and Soil Sciences, P.O. Box 6108, College of Agriculture and Forestry, West \\ Virginia University, Morgantown, WV 26506-6108, USA
}

(Received 18 July 2013; Accepted 21 January 2014)

\begin{abstract}
A new genus, a new generic name and two new species of phyllocoptine mites feeding on Yang-na, or hairy-leaf apitong, Dipterocarpus alatus Roxb. ex G. Don (Dipterocarpaceae) are described. Kosacarus is described as a new genus with Proneotegonotus dipterocarpi Chandrapatya and Boczek, 2000 designated as the type species; a redescription of Kosacarus dipterocapi (Chandrapatya and Boczek, 2000) is provided, including the male. Kosacarus rayongi is described herein as a new species. Bangphracarus is described as a new genus with Bangphracarus alatus new species as its type species. The distribution of each mite species is also provided.
\end{abstract}

Key words: Eriophyidae, Kosacarus, Bangphracarus, Dipterocarpus alatus, taxonomy, Thailand

\section{INTRODUCTION}

Dipterocarpus alatus Roxb. ex G. Don (Yang-na or hairy-leaf Apitong; Family Dipterocarpaceae) is a medium-sized to fairly large tree of up to $40 \mathrm{~m}$ tall or more. It is native to both evergreen and deciduous forests and commonly found in Cambodia, Laos, Myanmar, Philippines and Viet Nam, including Thailand (Orwa et al., 2009). It is one of the most important timber species next to teak in Thailand. Chandrapatya and Boczek $(1993,2000)$ reported three species of eriophyid mites associated with this plant in Thailand. They were Aculus dipterocarpi (Boczek, 1993); Siracharus dipterocarpi Chandrapatya and Boczek, 2000 and Proneotegonotus dipterocarpi Chandrapatya and Boczek, 2000.

* Corresponding author: e-mail: agramc@ku.ac.th DOI: $10.2300 /$ acari.23.15 
In this paper we redescribe Proneotegonous dipterocarpi and place it in a new genus, Kosacarus, since the dorsal pedipalp genual seta $d$ is long and bifurcate, opisthosoma not evenly rounded dorsally and ventral basifemoral setae are present on both legs. These characters were misdiagnosed in the original description. In addition, two species of phyllocoptine mites collected from $D$. alatus were also described which belong to a new genus.

All specimens were cleared in Keifer's I solution and mounted on slides using Keifer's III solution as the mounting medium. All terminology and measurements follow Lindquist (1996) and de Lillo et al. (2010). The measurements are given in micrometers $(\mu \mathrm{m})$ and are lengths or distances unless otherwise indicated. The number of specimens examined is given within parentheses. For females, each measurement of the holotype precedes the corresponding range for the paratypes and only the measurement of holotype is present when it is the same as paratypes. The count of dorsal annuli starts from the rear shield margin; the count of ventral annuli starts from the first lateral annulus at the prodorsal shield margin; the length of each leg is measured from the trochanteral base to the tip of tarsus, excluding empodium. All type specimens are deposited in the collection of the Department of Entomology, Kasetsart University, Bangkok, Thailand. Setal notation used in this paper follows Konvipasruang et al. (2012).

Setal notation used in this paper are as follows:

Prodorsal shield

sc scapular seta (dorsal setae)

Opisthosoma

c2 opisthosomal seta $c 2$ (lateral setae)

$d$ opisthosomal seta $d$ (1st ventral setae)

$e \quad$ opisthosomal seta $e$ (2nd ventral setae)

$f \quad$ opisthosomal seta $f$ (3rd ventral setae)

$h 1$ opisthosomal seta $h 1$ (accessory setae)

$h 2$ opisthosomal seta $h 2$ (caudal setae)

Pedipalp

ep pedipalp coxal seta (basal setae)

$d$ dorsal pedipalp genual seta (antapical setae)

$v \quad$ subapical pedipalp tarsal seta (sensory peg, papilla)

Setae on coxisternal plates

la proximal seta on coxisternum I (2nd coxal setae)

$1 b$ anterior seta on coxisternum I (1st coxal setae)

$2 a$ proximal seta on coxisternum II (3rd coxal setae)

$3 a$ proximal seta on coxisternum III (genital setae)

Legs

bv ventral basifemoral seta (femoral setae)

$l^{\prime \prime} \quad$ antaxial genual seta (patellar or genual setae)

$l^{\prime} \quad$ paraxial tibial seta (tibial setae)

$f t^{\prime}$ paraxial fastigial tarsal seta (dorsal tarsal setae)

$f t^{\prime \prime}$ antaxial fastigial tarsal seta (lateral tarsal setae)

$u^{\prime}$ paraxial unguinal tarsal seta (mesal setae) 


\title{
RESULTS
}

\author{
Family Eriophyidae Nalepa, 1898 \\ Subfamily Phyllocoptinae Nalepa, 1892 \\ Tribe Phyllocoptini Nalepa, 1892
}

Kosacarus Chandrapatya, Konvipasruang and Amrine gen. nov.

(Fig. 1)

Type species. Proneotegonotus dipterocarpi Chandrapatya and Boczek, 2000; Chandrapatya and Boczek, 2000. Bull. Pol. Acad. Sci., Biol. Sci. 48: 218, 221-223, f. 4.

Description. Female. Body fusiform. Gnathosoma projecting downwards, dorsal pedipalp genual seta $(d)$ moderately long and bifurcate. Prodorsal shield subrectangular, not much wider than opisthosoma, with thick and rounded prodorsal shield frontal lobe over gnathosoma; scapular tubercles plicate, axes longitudinal, situated centrally ahead of rear shield margin, scapular setae (sc) directed centrally. Paraxial tibial setae $\left(l^{\prime}\right)$ on leg I and antaxial genual seta $\left(l^{\prime \prime}\right)$ on leg II absent; empodium entire. Coxisterna I and II smooth, with all setae present; internal coxisternal apodeme absent. Dorsal annuli forming two subdorsal longitudinal ridges, first annulus incomplete, second and third annuli fused forming a broad plate, fourth annulus free. Opisthosomal setae $d$ and $e$ absent. Genital coverflap with several longitudinal ridges.

Diagnosis. This new genus is close to Neodicrothrix Mohanasundaram, 1984; but can be distinguished by the appearance of dorsal opisthosoma with subdorsal lateral ridges and ventral annuli with microtubercles present, different size of prodorsal shield, and internal coxisternal apodeme absent. Kosacarus has two subdorsal longitudinal ridges on dorsal opisthosoma; dorsal annuli smooth, ventral annuli microtuberculated; prodorsal shield not much wider than opisthosoma; internal coxisternal apodeme absent. Opisthosoma of Neodicrothrix evenly rounded, both dorsal and ventral annuli smooth, subequal dorso-ventrally; prodorsal shield conspicuously wider than opisthosoma and the internal coxisternal apodeme present.

To date, four Neodicrothrix species are known: Neodicrothrix tiliacorae Mohanasundaram, 1984; N. piperae Mohanasundaram, 1989; N. celloshieldae Umpathy, 1999 and N. rutacevagrans Umpathy, 1999. Neodicrothrix tiliacorae is the type species of the genus. However, after comparing all characters among these four species, it was found that $N$. rutacevagrans should be removed from this genus to the new genus proposed for this particular species due to the presence of seta $d$ on the opisthosoma and the absence of the dorsal pedipalp genual seta. Neodicrothrix celloshieldae is different from the type species in that there is a central longitudinal furrow on the dorsal opisthosoma (evenly rounded in others) and the internal coxisternal apodeme is not apparent. In addition, N. piperae is different from the type species in that the ventral annuli have faint microtubercles instead of a smooth surface as described in other Neodicrothrix spp.

Etymology. The generic name is after the Thai taxonomist, Associate Professor Kosol Charoensom of Kasetsart University, Bangkok, Thailand (Kosa- + acarus, a Latin word for mite).

Gender. Masculine. 
Kosacarus dipterocarpi (Chandrapatya and Boczek, 2000) Chandrapatya, Konvipasruang and Amrine

\section{(Fig. 1)}

Redescription. Female (n=9). Body fusiform, 122 (110-122) long, 58 (54-59) wide, 53 (53-55) thick; color in life light yellow. Gnathosoma 21 (20-24) long, projecting downwards; pedipalp coxal seta ep (2-3) long, dorsal pedipalp genual seta $d 8$ (6-10) long, bifurcate. Chelicerae 16 (1519) long, almost straight. Prodorsal shield 47 (44-48) long, 58 (54-59) wide, subrectangular, not much wider than opisthosoma, with thick and rounded prodorsal shield frontal lobe projecting over gnathosoma, 4 (4-6) long; prodorsal shield frontal lobe with an arc of thick granules extending back onto the shield. Prodorsal shield design with a strong marginal line, which curves forward and is truncate across the shield anteriorly, curving back on each side to the scapular tubercles and extending to near the rear shield margin; may be wax bearing line. Several short to moderate irregular longitudinal lines present centrally, becoming oblique near the margins.

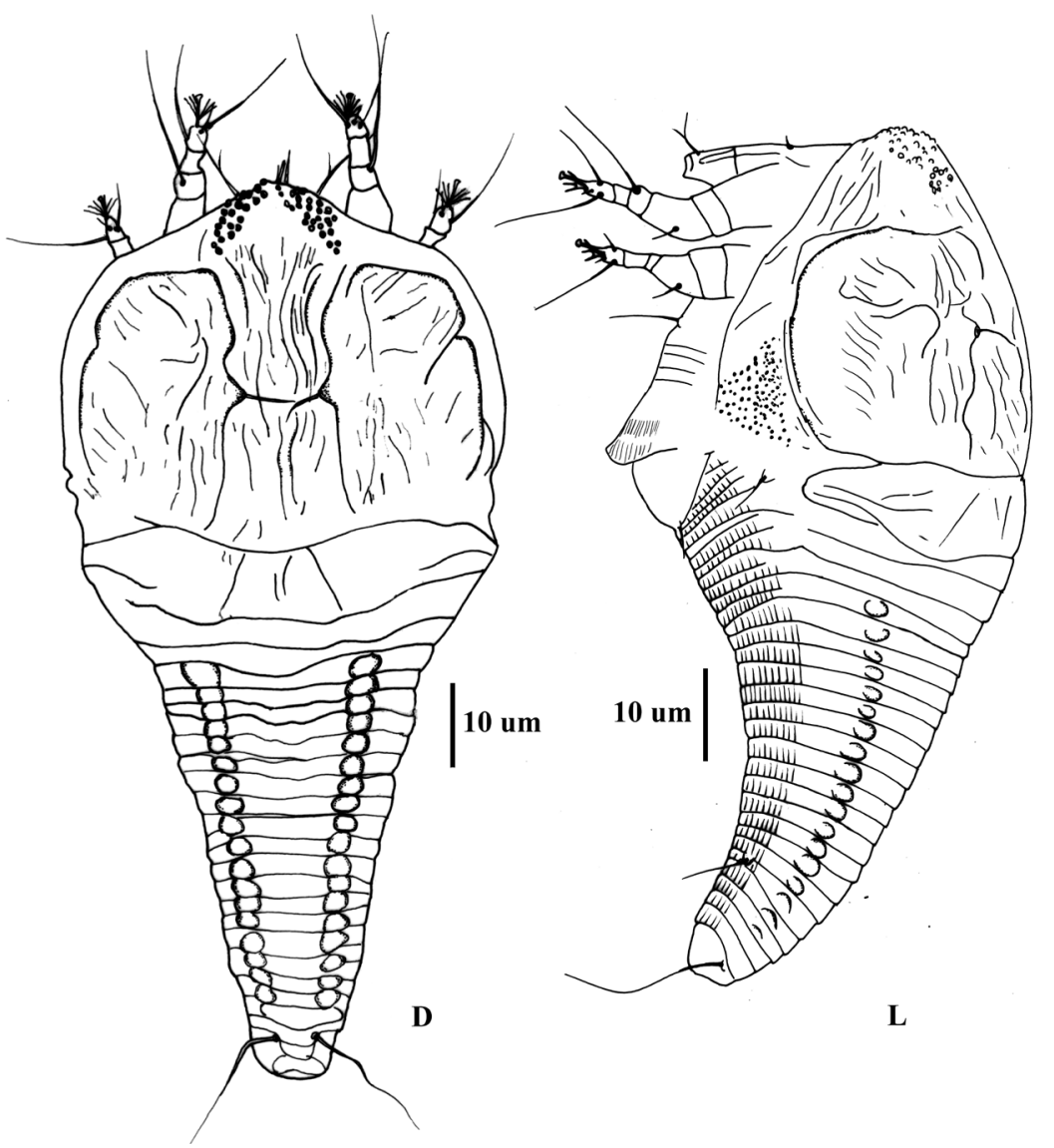

Fig. 1. Kosacarus dipterocarpi (Chandrapatya and Boczek, 2000) Chandrapatya, Konvipasruang and Amrine. Abbreviations: $\mathrm{D}=$ dorsal view of female; $\mathrm{L}=$ lateral view of female. 
Numerous irregular short, curved lines present on the lateral shield surface. A cluster of granules are located postero-laterally below the prodorsal shield. Scapular tubercles situated centrally, 19 (18-19) ahead of rear shield margin, plicate, 16 (11-16) apart. Scapular setae sc 7 (7-10) long, directed centrally. Leg I 19 (17-21) long; femur 7 (6-8) long, ventral basifemoral seta $b v 5$ (5-6) long; genu 3 (2-4) long, antaxial genual seta $l^{\prime \prime} 14$ (13-19) long; tibia 3 (2-3) long, paraxial tibial seta $l^{\prime}$ absent; tarsus 4 (3-4) long, antaxial fastigial tarsal seta $f t^{\prime \prime} 13$ (13-15) long, paraxial fastigial tarsal seta $f t^{\prime} 11$ (11-14) long, paraxial unguinal tarsal seta $u^{\prime} 3$ (2-4) long, solenidion 4 (3-4) long, straight, knobbed; empodium 3 (3-4) long, entire, 3-rayed. Leg II 16 (15-18) long;

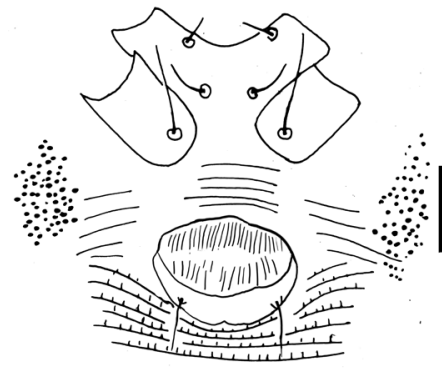

GF
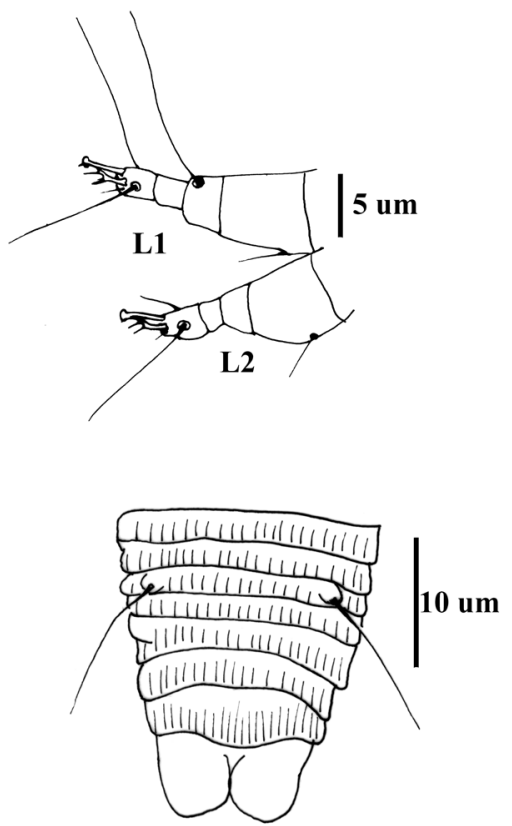

CV
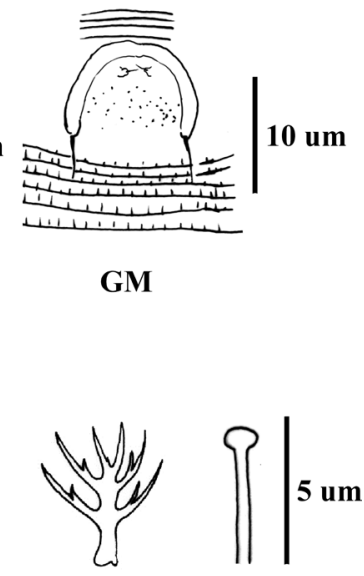

$\mathbf{E}$

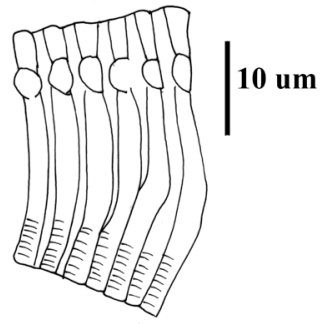

ES

Fig. 1. (continued). Kosacarus dipterocarpi (Chandrapatya and Boczek, 2000) Chandrapatya, Konvipasruang and Amrine. Abbreviations: $\mathrm{CV}=$ ventral view of cauda; E, empodium; $\mathrm{ES}=$ side skin of structure; $\mathrm{GF}=$ female genitalia; $\mathrm{GM}=$ male genitalia; $\mathrm{L}_{1}=\operatorname{leg} \mathrm{I} ; \mathrm{L}_{2}=\operatorname{leg} \mathrm{II} ; \mathrm{S}=$ solenidion. 
femur 6 (6-7) long, ventral basifemoral seta $b v 4$ (3-5) long; genu 3 (3-4) long, antaxial genual seta $l$ " absent; tibia 3 (2-3) long; tarsus 4 (3-4) long, antaxial fastigial tarsal seta $f t^{\prime \prime} 10$ (9-14) long, paraxial fastigial tarsal seta $f t^{\prime} 3$ (3-5) long, paraxial unguinal tarsal seta $u^{\prime} 3$ (2-3) long, solenidion 3 (2-3) long, as in leg I; empodium 3 (2-4) long, entire, 3-rayed. Coxisternal plates smooth. Internal coxisternal apodeme absent. Anterior seta on coxisternum I $1 b 4$ (4-5) long, 8 (5-8) apart; proximal seta on coxisternum I la 7 (6-8) long, 7 (5-9) apart; proximal seta on coxisternum II $2 a 12$ (12-14) long, 14 (8-13) apart; distance between setae $1 b$ and $1 a 6$ (5-7) long; coxigenital region with 4 (4-5) semiannuli, smooth. Opisthosoma with first dorsal annulus incomplete, expanded, covering the next two annuli, second and third annuli fused, forming a broad plate, fourth annulus free; followed by 20 (20-24) broad, smooth dorsal annuli forming two subdorsal longitudinal ridges, extending beyond opisthosomal seta $f$; annuli at each ridge with a circular demarcated flat disc or flattened knob. Ventral opisthosoma with 32 (32-36) annuli, with elongate microtubercles extending fully between margins of each annulus. Opisthosomal seta $c 2$ 8 (8-13) long, 43(38-44) apart, on annulus 7 (5-8); opisthosomal setae $d$ and $e$ absent; opisthosomal seta $f 10$ (10-16) long, 12 (10-13) apart, situated on annulus 27 (27-31) or annulus 5 from the rear. Opisthosomal seta $h_{2} 20$ (16-22) long; opisthosomal seta $h_{l}$ absent. Genital coverflap 19 (1419) wide, 16 (14-16) long, with about 15 longitudinal ridges on distal coverflap and 21 smaller, irregular-length longitudinal ridges on the basal coverflap; proximal seta on coxisternum III $3 a 4$ (3-4) long, 13 (11-15) apart. Several granules located laterad to the coxigenital annuli.

Male $(\mathrm{n}=2)$. Smaller than female, 103-120 long, 44 (49-60) wide, 50 thick. Gnathosoma 1820 long; pedipalp coxal seta ep 2-3 long, dorsal pedipalp genual seta $d$ 6-8 long, bifurcate. Chelicerae 16-17 long. Prodorsal shield 44-48 long, 48-58 wide, similar to female. Scapular tubercles situated 17 centrally ahead of rear shield margin, plicate, 14 apart. Scapular setae sc 6 long, directing centrally. Prodorsal shield frontal lobe 4-6 long. Leg I 18-19 long; femur 7-9 long, ventral basifemoral seta $b v$ 5-6 long; genu 3-4 long, antaxial genual seta $l$ " 15-17 long; tibia 3-4 long, paraxial tibial seta $l^{\prime}$ absent; tarsus 4 long, antaxial fastigial tarsal seta $f t^{\prime \prime} 13-15$ long, paraxial fastigial tarsal seta $f t^{\prime}$ 12-14 long, paraxial unguinal tarsal seta $u^{\prime}$ 2-3 long, solenidion 4 long, straight, knobbed; empodium 3 long, entire, 3-rayed. Leg II 16-17 long; femur 6-7 long, ventral basifemoral seta $b v 4$ long; genu 3-4 long, antaxial genual seta $l$ " absent; tibia 3-4 long; tarsus 3-4 long, antaxial fastigial tarsal seta $f t^{\prime \prime} 12-14$ long, paraxial fastigial tarsal seta $f t^{\prime} 3$ long, paraxial unguinal tarsal seta $u^{\prime}$ 2-3 long, solenidion 3-4 long, as in leg I; empodium 3 long, entire, 3 -rayed. Coxisternal plates smooth. Internal coxisternal apodeme absent. Anterior seta on coxisternum I $1 b 3$ long, 7 apart; proximal seta on coxisternum I $1 a 10$ long, 7-8 apart; proximal seta on coxisternum II $2 a$ 12-13 long, 12-14 apart; distance between setae $1 b$ and $1 a$ 6-7 long; coxigenital region with 4 semiannuli, smooth. Opisthosoma with 26 dorsal annuli, 29-31 ventral annuli. Opisthosomal seta $c 2$ 10-13 long, 34-55 apart, on annulus 5-6; opisthosomal setae $d$ and $e$ absent; opisthosomal seta $f$ 10-14 long, 12-13 apart, situated laterally on annulus 25-26 or annulus 5 from the rear. Opisthosomal seta $h_{2} 18$ long; opisthosomal seta $h_{l}$ absent. Genitalia 1315 wide; proximal seta on coxisternum III $3 a 5$ long, 14-15 apart.

Etymology. The species name is based on the host plant generic name, Dipterocarpus.

Type material. Female holotype, paratypes and male paratype on 27 slides labeled \# 943.5.

Host plant and locality. Collected from leaves of Dipterocarpus alatus Roxb. ex G. Don 
(Yang-na, or hairy-leaf Apitong), Dipterocarpaceae; Amphoe Si Racha, Chon Buri Province, Thailand (Latitude N: 13.167 Longitude E: 100.933), 13 September 1998, coll. Chandrapatya.

Relation to host. Vagrant on the lower leaf surface. No apparent damage was observed.

Kosacarus rayongi Chandrapatya, Konvipasruang and Amrine sp. nov.

(Fig. 2)

Description. Female (n=4). Body fusiform, 138 (138-149) long, 71 (71-74) wide, 62 (62-69) thick; color in life light yellow. Gnathosoma 22 (22-24) long, projecting downwards; pedipalp coxal seta ep 3 (3-4) long, dorsal pedipalp genual seta $d 13$ (10-13) long, bifurcate. Chelicerae 20 (19-21) long, almost straight. Prodorsal shield 56 (53-57) long, 71 (69-73) wide, subrectangular, not much wider than anterior opisthosoma. Shield design consists of 4 hump-like closed cells on each side, numerous curved lines are associated with each cell. Two submedian lines form a central bulbous shape posteriorly, on which are numerous, short irregular lines. Prodorsal shield

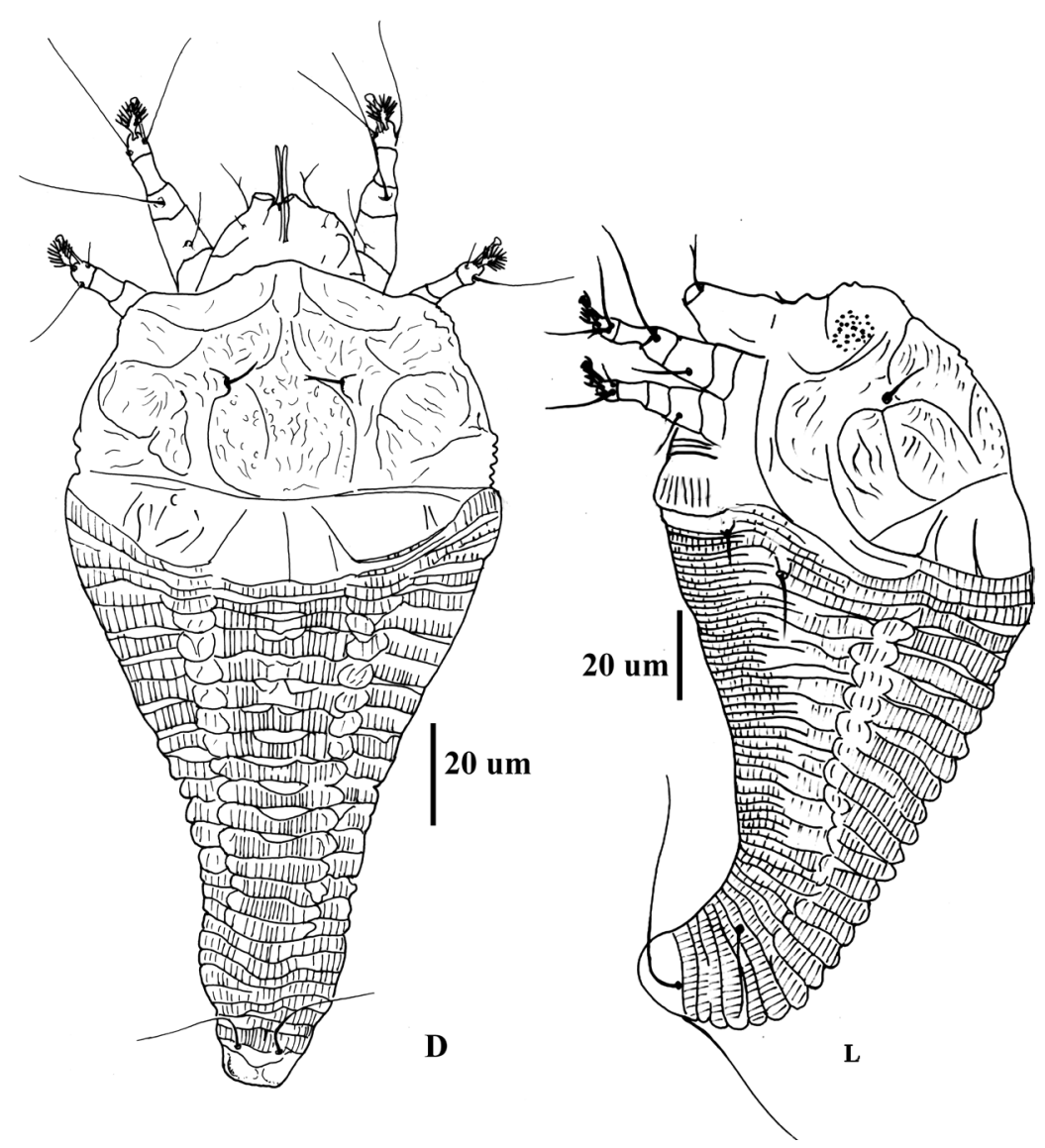

Fig. 2. Kosacarus rayongi Chandrapatya, Konvipasruang and Amrine sp. nov. Abbreviations are same as in Fig. 1. 

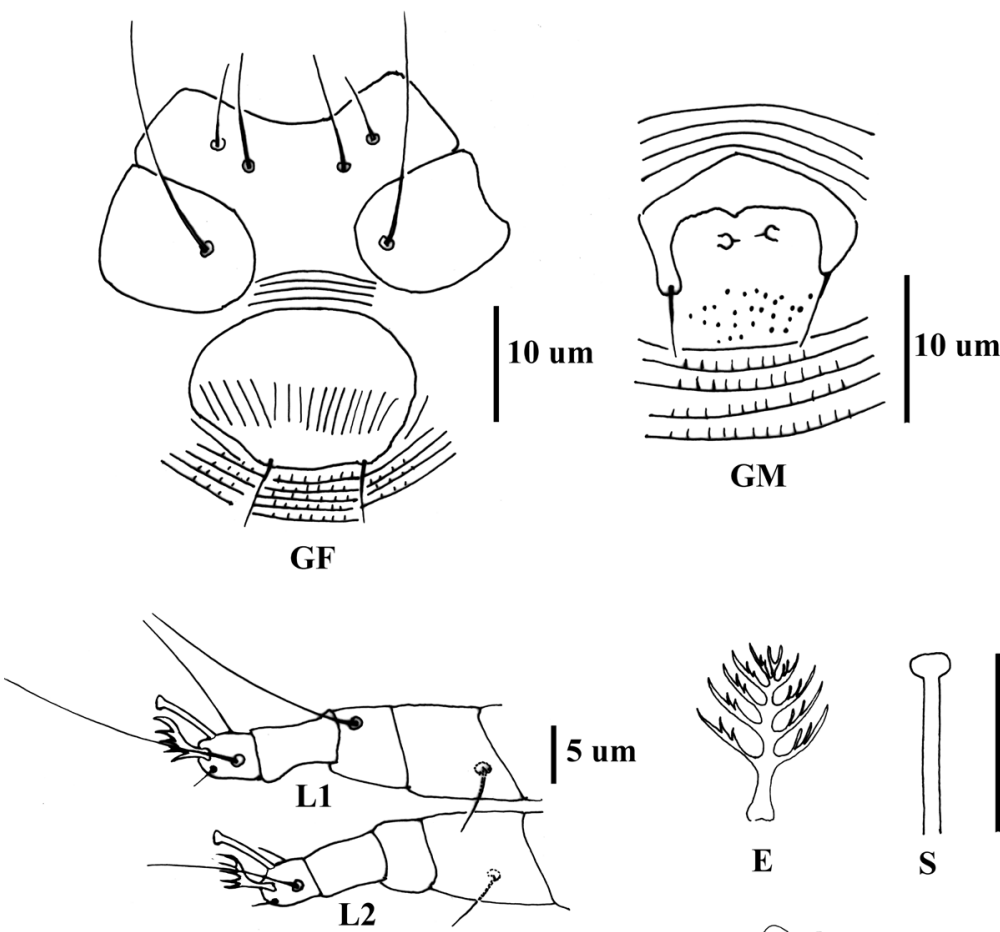

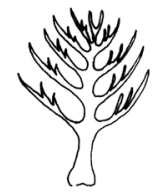

$\mathbf{E}$
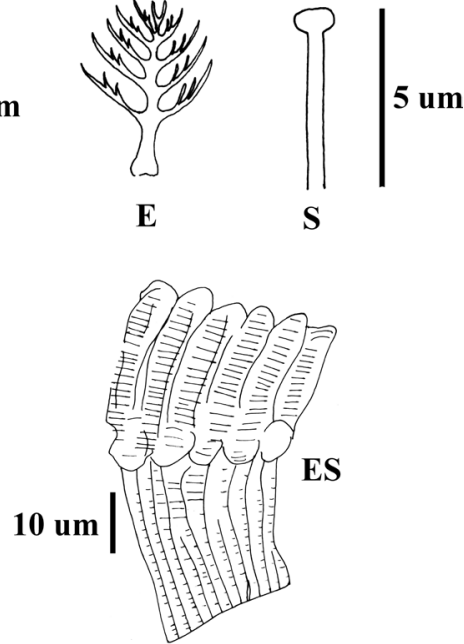

Fig. 2. (continued). Kosacarus rayongi Chandrapatya, Konvipasruang and Amrine sp. nov. Abbreviations are same as in Fig. 1.

frontal lobe round, 6 (5-6) long, slightly dense, with several thick granules on each side. Scapular tubercles situated centrally, 31(27-33) ahead of rear shield margin, bulbous, 22 (22-26) apart. Scapular setae sc 9 (9-11), fine, directed centrally. Leg I 27 (27-32) long; femur 12 (11-13) long, ventral basifemoral seta $b v 10$ (10-12) long; genu 5 (5-6) long, antaxial genual seta $l^{\prime \prime} 21$ (20-23) long; tibia 8 (8-9) long, paraxial tibial seta $l^{\prime}$ absent; tarsus 4 (4-5) long, antaxial fastigial tarsal seta $f t^{\prime \prime} 22$ (19-23) long, paraxial fastigial tarsal seta $f t^{\prime} 14$ (13-18) long, paraxial unguinal tarsal seta $u^{\prime} 2$ (2-3) long, solenidion 6 (5-6) long, straight, knobbed; empodium 5 (5-6) long, entire, 6-rayed. Leg II 26 (24-27) long; femur 9 (8-10) long, ventral basifemoral seta bv 11 (10-11) long; genu 5 (5-6) long, antaxial genual seta $l^{\prime \prime}$ absent; tibia 7 (6-8) long; tarsus 4 (4-5) long, antaxial 
fastigial tarsal seta $f t^{\prime \prime} 17$ (17-19) long, paraxial fastigial tarsal seta $f t^{\prime} 4$ (4-5) long, paraxial unguinal tarsal seta $u^{\prime} 3$ (2-3) long, solenidion 6 (5-6) long, as in leg I; empodium 5(4-6) long, entire, 6-rayed. Coxisternal plates smooth. Internal coxisternal apodeme absent. Anterior seta on coxisternum I $1 b 6$ (6-8) long, 14 (14-16) apart; proximal seta on coxisternum I $1 a 10$ (9-13) long, 9 (9-10) apart; proximal seta on coxisternum II $2 a 22$ (22-33) long, 14 (14-17) apart; distance between setae $1 b$ and $1 a 4$ (3-5) long; coxigenital region with 4 (4-5) semiannuli, smooth. Opisthosoma with first dorsal annulus expanded and plate-like, fused with prodorsal shield and followed by about 26 (26-32) broad dorsal annuli; ventrally with 50 (44-52) annuli. Dorsal annuli with a row of elongate microtubercles extending between margins of each annulus, ventral annuli with elongate microtubercles situated on rear margin of each annulus. Dorsal annuli forming two subdorsal longitudinal ridges with oval, knob-like structures, all ridges fading before opisthosomal seta $f$. Opisthosomal seta $c 212$ (12-18) long, 41 (41-50) apart, on annulus 5 (5-8); opisthosomal setae $d$ and $e$ absent; opisthosomal seta $f 14$ (13-16) long, 13 (13-15) apart, situated postero-ventrally on annulus 44 (38-46) or annulus 6 (5-6) from the rear. Opisthosomal seta $h_{2} 37$ (35-37) long; opisthosomal seta $h_{l}$ absent. Genital coverflap 22 (18-22) wide, 17 (1619) long, with 16 (15-18) longitudinal ridges; proximal seta on coxisternum III $3 a 6$ (6-7) long, $10(8-11)$ apart.

Male $(\mathrm{n}=4)$. Body fusiform, 132-142 long, 62-71 wide. Gnathosoma 20-22 long; pedipalp coxal seta ep 2-3 long, dorsal pedipalp genual seta $d$ 11-12 long, bifurcate. Chelicerae 20-22 long. Prodorsal shield 53-56 long, 62-70 wide; similar to female. Prodorsal shield frontal lobe 6-7 long. Scapular tubercles situated 30-32 centrally ahead of rear shield margin, bulbous, 16-22 apart. Scapular setae sc 9-10, fine, directing centrally. Leg I 24-29 long; femur 10-12 long, ventral basifemoral seta $b v$ 9-11 long; genu 5-7 long, antaxial genual seta $l^{\prime \prime} 16-19$ long; tibia 6-7 long, paraxial tibial seta $l^{\prime}$ absent; tarsus 4-5 long, antaxial fastigial tarsal seta $f t^{\prime \prime}$ 17-20 long, paraxial fastigial tarsal seta $f^{\prime}$ 15-18 long, paraxial unguinal tarsal seta $u^{\prime}$ 2-3 long, solenidion 5-6 long, straight, knobbed; empodium 4-6 long, entire, 6-rayed. Leg II 23-24 long; femur 8-10 long, ventral basifemoral seta $b v$ 8-11 long; genu 5-6 long, antaxial genual seta $l^{\prime \prime}$ absent; tibia 5-6 long; tarsus 4-5 long, antaxial fastigial tarsal seta $\mathrm{ft}^{\prime \prime}$ 16-19 long, paraxial fastigial tarsal seta $\mathrm{ft}^{\prime}$ 4-5 long, paraxial unguinal tarsal seta $u^{\prime}$ 2-4 long, solenidion 5-6 long, as in leg I; empodium 5-6 long, entire, 6-rayed. Coxisternal plates smooth. Internal coxisternal apodeme absent. Anterior seta on coxisternum I 1b 7-8 long, 13-14 apart; proximal seta on coxisternum I 1a 10-13 long, 8-10 apart; proximal seta on coxisternum II $2 a$ 20-27 long, 15-16 apart; distance between seta $1 b$ and 1a 3-4 long; coxigenital region with 4-5 semiannuli, smooth. Opisthosoma: with 26-29 dorsal annuli, 41-49 ventral annuli. Opisthosomal seta $c 2$ 13-18 long, 37-48 apart, on annulus 5-6; opisthosomal setae $d$ and $e$ absent; opisthosomal seta $f$ 12-18 long, 13-14 apart, situated on annulus 32-42 or annulus 5-6 from the rear. Opisthosomal seta $h_{2}$ 24-34 long; opisthosomal seta $h_{l}$ absent. Genitalia 16-18 wide; proximal seta on coxisternum III $3 a$ 6-8 long, 9-11 apart.

Etymology. The species name is based on the location, Rayong province, where the mite was collected.

Type material. Female holotype, paratypes and male paratypes on 8 slides labeled \# 1684.2.

Host plant and locality. Collected from leaves of Dipterocarpus alatus Roxb. ex G. Don (Yang-na), Dipterocarpaceae; Amphoe Mueang, Rayong Province, Thailand (Latitude N: 12 41' 
00", Longitude E: 101 17' 00"), 12 October 1999, coll. A. Chandrapatya.

Relation to host. Vagrant on the lower leaf surface. No apparent damage was observed.

Diagnosis. This mite species can be distinguished from Kosacarus dipterocarpi by differences in shield pattern, number of empodial rays, dorsal annuli, ridges and structure of the coverflap. Shield pattern of $K$. dipterocarp $i$ with one long curved line laterally and several curved lines on the whole shield surface; empodium 3-rayed; dorsal annuli smooth, forming two subdorsal longitudinal ridges extending beyond opisthosomal seta $f$; coverflap with 15-21 longitudinal ridges arranged in two ranks. Shield pattern of the new species with four hump-like closed cells on each side and numerous curved lines are associated with each cell, two submedian lines forming central bulbous shape posteriorly, bearing numerous irregular short lines; empodium 6-rayed; dorsal annuli with elongated microtubercles, forming two subdorsal longitudinal ridges fading before opisthosomal seta $f$; distal coverflap with a row of 15-18 longitudinal ridges.

Bangphracarus Chandrapatya, Konvipasruang and Amrine gen. nov.

(Fig. 3)

Type species: Bangphracarus alatus sp. nov.

Description. Female. Body fusiform. Gnathosoma projecting downwards, dorsal pedipalp genual seta $d$ moderately long and bifurcate. Prodorsal shield subspherical, longer than wide, conspicuously wider than opisthosoma; prodorsal shield frontal lobe thick and broad over gnathosoma; scapular setae $s c$ stout and curved, thorn-like, on large scapular tubercles, situated well ahead of rear shield margin, scapular setae $s c$ directed to the rear. Paraxial tibial seta $l^{\prime}$ on leg I and antaxial genual seta $l$ " on leg II absent; empodium entire. Coxisterna I and II with all setae present. Dorsal opisthosoma with short and narrow middorsal furrow, two subdorsal ridges join posteriorly, three annuli anterior to opisthosomal setae $f$ forming a short middorsal ridge. First - fifth dorsal annuli curved upwards, forming posterior vertical wall of prodorsal shield. Dorsal annuli smooth. Opisthosomal setae $d$ and $e$ absent. Genital coverflap smooth.

Diagnosis. This new genus is close to Neodicrothrix but it can be distinguished by the distinctive shape of the prodorsal shield and scapular setae, opisthosoma and annuli. Prodorsal shield of Bangphracarus is subspherical, longer than wide, with curved, stout scapular setae directed posteriorly. Prodorsal shield of Neodicrothrix is subrectangular, scapular setae $s c$ of Neodicrothrix is straight and directed centrally. Dorsal opisthosoma of Bangphracarus with short and narrow middorsal furrow, bordered by subdorsal longitudinal ridges; first to fifth dorsal annuli expanded, curved upwards, forming posterior vertical wall of prodorsal shield; dorsal annuli smooth, ventral annuli microtuberculated. Opisthosoma of Neodicrothrix evenly rounded, dorsal and ventral annuli smooth.

Etymology. The generic name is after the location (Bang Phra- + acarus, a Latin word for mite) where this mite was collected.

Gender. Masculine.

Bangphracarus alatus Chandrapatya, Konvipasruang and Amrine sp. nov.

(Fig. 3)

Description. Female (n=13). Body fusiform, 139 (129-146) long, 68 (62-70) wide, 69-72 


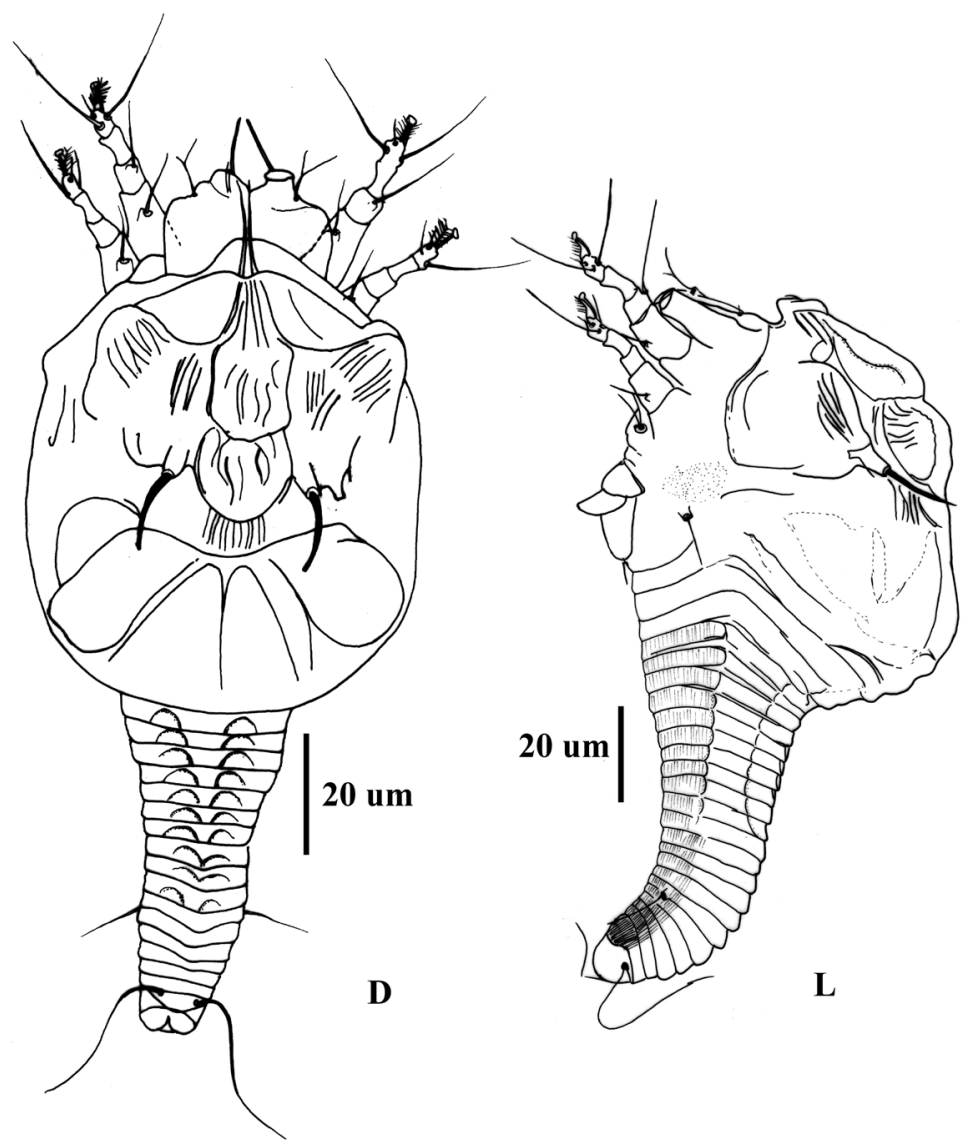

Fig. 3. Bangphracarus alatus Chandrapatya, Konvipasruang and Amrine sp. nov. Abbreviations are same as in Fig. 1.

thick, color in life light red. Gnathosoma 26 (26-31) long, projecting downwards; pedipalp coxal seta ep 2 (2-3) long, dorsal pedipalp genual seta $d 8$ (7-11) long, bifurcate. Chelicerae 20 (19-23) long, almost straight. Prodorsal shield 82 (78-88) long, 68 (61-69) wide. Prodorsal shield conspicuously wider than opisthosoma. Shield design consists of short median and admedian lines of the same length, extending back 1/5 from anterior margin, paired submedian lines 1 forming a vague figure- 8 between, and anterior to, scapular setae, submedian lines 2 running laterally around a large hump-like structure on each side. Several curved and straight lines occur on the central part of the shield on, and behind the bulbous structures. Prodorsal shield frontal lobe thick and round anteriorly, projecting over gnathosoma, 4 (3-4) long. Scapular tubercles situated 42 (43-44) ahead of rear shield margin, 25 (25-36) apart. Scapular setae sc 14 (13-15) long, stout and curve, thorn-like, directed to the rear. Leg I 30 (27-30) long; femur 11 (10-12) long, ventral basifemoral seta $b v 8$ (8-11) long; genu 5 (5-6) long, antaxial genual seta $l^{\prime \prime} 20$ (2023) long; tibia 6 (6-7) long, paraxial tibial seta $l^{\prime}$ absent; tarsus 5 (4-5) long, antaxial fastigial 

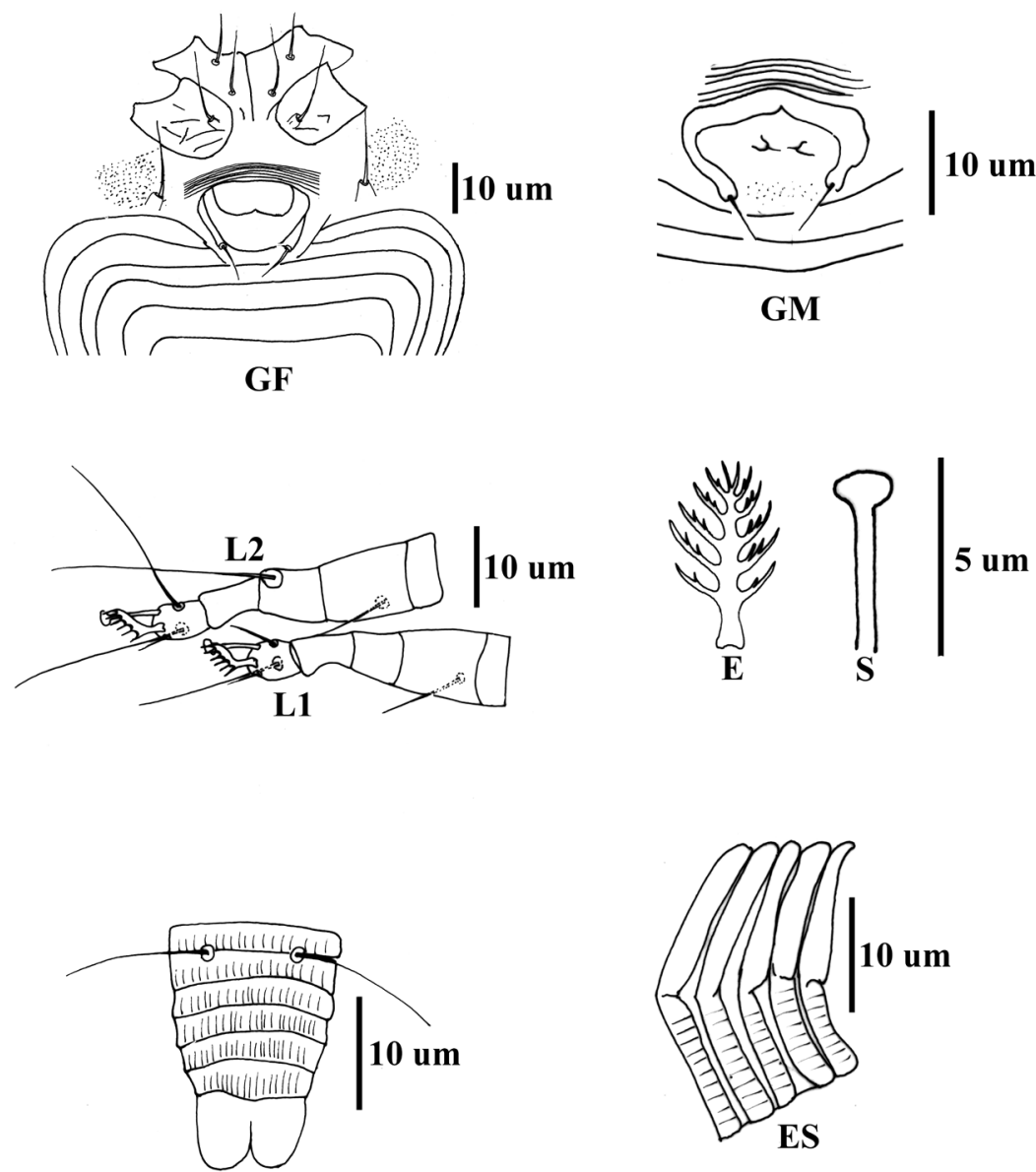

CV

Fig. 3. (continued). Bangphracarus alatus Chandrapatya, Konvipasruang and Amrine sp. nov.

Abbreviations are same as in Fig. 1.

tarsal seta $f t^{\prime \prime} 19$ (18-20) long, paraxial fastigial tarsal seta $f t^{\prime} 17$ (16-19) long, paraxial unguinal tarsal seta $u^{\prime} 3$ (3-5) long, solenidion 4 (4-7) long, straight, knobbed; empodium 4 (4-6) long, entire, 6-rayed. Leg II 26 (24-28) long; femur 10 (8-11) long, ventral basifemoral seta $b v 9$ (8-10) long; genu 4 (4-6) long, antaxial genual seta $l^{\prime \prime}$ absent; tibia 6 (4-5) long; tarsus 4 (4-5) long, antaxial fastigial tarsal seta $f t^{\prime \prime} 16$ (16-18) long, paraxial fastigial tarsal seta $f t^{\prime} 4$ (4-5) long, paraxial unguinal tarsal seta $u^{\prime} 3$ (3-4) long, solenidion (5-6) long, as in leg I, 5; empodium 4 (4-6) long, entire, 6-rayed. Coxisternal plate I smooth, coxisternal plate II with a few short lines. Internal coxisternal apodeme a line 12 (10-14) long. Anterior seta on coxisternum I $1 b 7$ (7-9) long, 13 (11-14) apart; proximal seta on coxisternum I 1 a 10 (10-14) long, 7 (6-8) apart; proximal seta on coxisternum II $2 a 14$ (14-18) long, 15 (13-17) apart; distance between setae $1 b$ and $1 a 5$ (5-7) long; coxigenital region with 8 (8-9) semiannuli, smooth. Opisthosoma with 22 (22-25) 
broad smooth dorsal annuli. First to fifth dorsal annuli expanded, $5^{\text {th }}-14^{\text {th }}$ annuli forming two subdorsal longitudinal ridges, with short and narrow furrow between subdorsal ridges, subdorsal ridges joined posteriorly above opisthosomal setae $f$ and forming short central ridge. First -fifth dorsal annuli forming posterior wall wrapped around posterior margin of prodorsal shield. Ventral opisthosoma with 24 (24-32) annuli, with elongate microtubercles situated on rear margin of each annulus. The last 5-6 ventral annuli with elongated microtubercles. Opisthosomal seta $c 2$ 10 (10-13) long, 38 (33-40) apart, on annulus 2 (1-2); opisthosomal setae $d$ and $e$ absent; opisthosomal seta $f 13$ (13-15) long, 10 (9-11) apart, situated postero-ventrally on annulus 18 (18-26) or annulus 6 (5-6) from the rear. Opisthosomal seta $h_{2} 22$ (22-30) long; opisthosomal seta $h_{l}$ absent. Genital coverflap 24 (20-30) wide, 17 (17-22) long; proximal seta on coxisternum III $3 a$ a (6-8) long, 14 (12-14) apart; genital coverflap smooth.

Male $(\mathrm{n}=2)$. Smaller than female, 109-124 long, 51-55 wide. Gnathosoma 25 long; pedipalp coxal seta ep 2-3 long, dorsal pedipalp genual seta $d$ 9-11 long, bifurcate. Chelicerae 20-21 long. Prodorsal shield 61-65 long, 53-55 wide; ornamentation as in the female. Prodorsal shield frontal lobe 4-5 long. Scapular tubercles situated 41-43 ahead of rear shield margin, 25-30 apart. Scapular setae sc 11-12 long, stout and curve, thorn-like, directed to the rear. Leg I 24-28 long; femur 10-11 long, ventral basifemoral seta $b v$ 8-9 long; genu 4 long, antaxial genual seta $l$ " 19-22 long; tibia 6-7 long, paraxial tibial seta $l^{\prime}$ absent; tarsus 4-5 long, antaxial fastigial tarsal seta $\mathrm{ft}^{\prime \prime}$ 18-19 long, paraxial fastigial tarsal seta $f t^{\prime} 12-14$ long, paraxial unguinal tarsal seta $u^{\prime} 3-4$ long, solenidion 5-6 long, straight, knobbed; empodium 4-5 long, entire, 6-rayed. Leg II 21-23 long; femur 9-10 long, ventral basifemoral seta $b v$ 8-9 long; genu 4-5 long, antaxial genual seta $l$ " absent; tibia 4-5 long; tarsus 4-5 long, antaxial fastigial tarsal seta $f t^{\prime \prime}$ 16-25 long, paraxial fastigial tarsal seta $f t^{\prime} 3-4$ long, paraxial unguinal tarsal seta $u^{\prime} 2-3$ long, solenidion 5 long, as in leg I; empodium 4-5 long, entire, 6-rayed. Coxisternal plate I smooth, coxisternal plate II with a few short lines. Internal coxisternal apodeme a line 11-13 long. Anterior seta on coxisternum I $1 b$ 7-8 long, 11-12 apart; proximal seta on coxisternum I 1a 9-11 long, 5-6 apart; proximal seta on coxisternum II $2 a$ 16-19 long, 13-14 apart; distance between setae $1 b$ and $1 a 6$ long. coxigenital region with 5-6 semiannuli, smooth. Opisthosoma with 27-28 dorsal annuli, 26-30 ventral annuli. Opisthosomal seta $c 2$ 8-11 long, 28-32 apart, on annulus 1-2; opisthosomal setae $d$ and $e$ absent; opisthosomal seta $f$ 12-14 long, 9-10 apart, situated on annulus 21-24 or annulus 5-6 from the rear. Opisthosomal seta $h_{2}$ 27-29 long; opisthosomal seta $h_{1}$ absent. Genitalia 14-16 wide, proximal seta on coxisternum III 3 a 5-9 long, 9-11 apart.

Etymology. The species name is based on the host plant species name, alatus.

Type material. Female holotype, paratypes and male paratypes on 24 slides labeled \# 1182; female paratypes on 12 slides labeled \# 1684.1.

Host plant and locality. Collected from leaves of Dipterocarpus alatus Roxb. ex G. Don (Yang-na), Dipterocarpaceae; Tombon Bang Phra, Amphoe Si Racha, Chon Buri Province, Thailand (Latitude N: 13 12' 00" Longitude E: 100 56' 00"), 19 February 1999, coll. A. Chandrapatya (\#1182) and Amphoe Mueang, Rayong Province, Thailand (Latitude N: 12 41' 00" Longitude E: 101 17' 00"), 12 October 1999, coll. A. Chandrapatya (\#1684.1).

Relation to host. Vagrant on the lower leaf surface. No apparent damage was observed. 


\section{ACKNOWLEDGEMENTS}

A part of this research was partially supported by the Faculty of Agriculture and the Center for Advanced Studies for Agriculture and Food, Institute for Advanced Studies, Kasetsart University, under the Higher Education Research Promotion and National Research University Project of Thailand, Office of the Higher Education Commission and Thailand Research Fund (TRF Senior Research Scholar \#RTA4880006).

\section{REFERENCES}

Amrine, J.W. Jr., T.A. Stasny and C.H.W. Flechtmann (2003) Revised Keys to World Genera of Eriophyoidea (Acari: Prostigmata). 244 p., Indira Publishing House, Michigan, U.S.A.

Chandrapatya, A. and J. Boczek (1993) Studies on eriophyid mites (Acari: Eriophyoidea). XII. Bulletin of the Polish Academy of Sciences, Biological Sciences, 41: 45-52.

Chandrapatya, A. and J. Boczek (2000) Studies on eriophyoid mites (Acari: Eriophyoidea). XXXIII. Bulletin of the Polish Academy of Sciences, Biological Sciences, 48: 211-223.

de Lillo, E., C. Craemer, J.W. Amrine Jr. and G. Nuzzaci (2010) Recommended procedures and techniques for morphological studies of Eriophyoidea (Acari: Prostigmata). Experimental and Applied Acarology, 51: 283-307.

Keifer, H. H. (1954) Eriophyid studies XXII. Bulletin of the California Department of Agriculture, 43: 121-131.

Konvipasruang, P., A. Chandrapatya and J.W. Amrine jr. (2012) Redescription of seven species of eriophyoid mite from bamboo (Poaceae, Bambuseae) in Thailand. Journal of the Acarology Society of Japan, 21: 67-94.

Lindquist E. E. (1996) External Anatomy and Notation of Structures. In: World Crop Pests, 6: Eriophyoid Mites: Their Biology, Natural Enemies and Control. (eds., Lindquist E.E., M.W. Sabelis and J. Bruin), pp. 3-31, Elsevier Science Publishing, Amsterdam,.

Mohanasundaram, M. (1984) New eriophyid mites from India (Acarina: Eriophyoidea). Oriental Insects, 18: 251-283.

Mohanasundaram, M. (1989) Four new species of Eriophyoidea (Acari) from Tamil Nadu. Entomon, 14: 261-267.

Orwa, C., A. Mutua, R. Kindt, R. Jamnadass and S. Anthony (2009) Dipterocarpus alatus Roxb. ex G. Don. Agroforestree Database: a tree reference and selection guide version 4. http:/www.worldagroforestry.org/treedb/AFTPDFS/ Dipterocarpus_alatus.pdf

Umapathy, G. (1999) Description of new species of eriophyid mites (Acari: Eriophyidae: Phyllocoptinae) from South India. Indian Journal of Acarology, 15: 99-105. 